\title{
Aplicación de herramientas de modelado molecular para evaluar las interacciones de inhibidores de la proteasa NS2B/NS3 del vi- rus del dengue tipo 2
}

\author{
Application of molecular modeling tools to evaluate the interaction par \\ protease inhibitors NS2B/NS3 dengue virus type 2
}

\author{
Muriel Díaz ${ }^{1}$, Javier Orozco ${ }^{2}$, Catalina Vivas-Gomez ${ }^{3}$, Ricardo Vivas-Reyes ${ }^{4}$, Alfredo Pérez-Gamboa ${ }^{5}$ \\ 'Química, Universidad de Cartagena, Programa de Química, Facultad de Ciencias Exactas y Naturales. \\ Grupo de Química Cuántica y Teórica. Cartagena, Colombia \\ 2 Químico, Universidad de Cartagena, Programa de Química, Facultad de Ciencias Exactas y Naturales. \\ Grupo de Química Cuántica y Teórica. Cartagena, Colombia \\ Médica, Universidad del Sinú, Cartagena, Programa de Química, Facultad de Ciencias Exactas y Naturales. \\ Grupo de Quimica Cuántica y Teórica. Cartagena, Colombia \\ ${ }^{4}$ Doctor en Ciencias Químicas. Universidad de Cartagena, Programa de Química, Facultad de Ciencias Exactas y Naturales. \\ Grupo de Quimica Cuántica y Teórica. Cartagena, Colombia. \\ Grupo Ginumec, Corporación Universitaria Rafael Núnez, Facultad de ciencias de la Salud. Programa de Medicina. Cartagena-Colombia. \\ 5 Doctor en Ciencias Químicas. Universidad del Atlántico, Programa de Química, Facultad de Ciencias Básicas. \\ Grupo de Investigación en Compuestos Heterocíclicos. Barranquilla, Colombia \\ rvivasr@unicartagena.edu.co
}

Cite this article as: M. Diaz, J. Orozco, Catalina Vivas-Gómez, R. Vivas-Reyes. A. Pérez-Gamboa. L. "Aplicación de herramientas de modelado molecular para evaluar las interacciones de inhibidores de la proteasa NS2B/NS3 del virus del dengue tipo 2", Prospectiva, Vol 18, N $1,53-59,2020$

Recibido: 23/08/2019 / Aceptado: 10/10/2019

https://doi.org/10.15665/rp.v18i1.2098

\begin{abstract}
RESUMEN
El dengue es una enfermedad viral endemo-epidémica más importante en términos de morbilidad y mortalidad. La proteasa NS3 es un objetivo atractivo para el desarrollo de nuevos inhibidores antivirales y su estructura tridimensional ha sido determinada, pero los determinantes necesarios para la activación de la enzima por el cofactor NS2B solo han sido caracterizados en forma limitada. Quince compuestos que corresponden a una serie de inhibidores de la proteasa NS2B/ NS3 del virus del dengue, con actividad inhibitoria in vitro comprobada, fueron sometidos a modelado molecular con docking y 3D-QSAR. Los péptidos que tienen sustituyentes más grandes en tamaño poseen actividades inhibitorias más altas que aquellos que no tienen grupos grandes. Se hacen recomendaciones de modificaciones estructurales derivadas del estudio 3D-QSAR para aumentar la actividad biológica de estos péptidos.
\end{abstract}

\begin{abstract}
Dengue is an important endemo-epidemic viral disease in terms of morbidity and mortality. The NS3 protease is an attractive target for the development of new antiviral inhibitors and its three-dimensional structure has been determined, but the determinants necessary for the activation of the enzyme by the NS2B cofactor have only been characterized to a limited extent. Fifteen compound corresponding to a series of inhibitors of the NS2B/NS3 protease of dengue virus, with proven in vitro inhibitory activity, were subjected to molecular modeling with docking and 3D-QSAR. Peptides that have larger substituents in size have higher inhibitory activities than those that do not have large groups. Recommendations for structural modifications derived from the 3D-QSAR study are made to increase the biologicala ctivity of these peptides.
\end{abstract}




\section{INTRODUCCIÓN}

El dengue es una enfermedad viral endemo-epidémica transmitida por un mosquito. Es una de las enfermedades virales más importantes propagadas por artrópodos, en términos de morbilidad y mortalidad, siendo el Aedes aegypti su principal vector [1].

La incidencia del dengue a nivel mundial se ha incrementado dramáticamente en el mundo, llegándose a estimar en las últimas décadas que existen cerca de2.500 millones de personas expuestas al dengue en el planeta, esto es alrededor de un cuarto de la población mundial. El dengue presenta una gran incidencia, en términos de morbilidad y mortalidad. La organización mundial de la salud estima que se presenta un estimado de aproximadamente entre 50 a 100 millones de infecciones anualmente alrededor del mundo cada año por causa de este virus del dengue que es el agente causal de la enfermedad conocida como dengue [2-6]. La meta de encontrar nuevos compuestos antivirales, dirigidos a blancos enzimáticos del dengue que son esenciales para la replicación viral, requiere el estudio estructural tridimensional detallado de las proteínas NS3 y NS5. Así, la proteasa NS3 del virus del dengue es un objetivo atractivo para el desarrollo de inhibidores potentes y selectivos en la terapia contra el virus del dengue [7-10].

La proteasa NS3 representa un blanco atractivo para el desarrollo de nuevos inhibidores antivirales. La estructura tridimensional de la NS3 ha sido determinada, pero los determinantes necesarios para la activación de la enzima por el cofactor NS2B solo han sido caracterizados en forma parcial [11].

El dominio de la proteasa del virus del dengue consiste de los primeros 170 aminoácidos de la NS3, presenta una homología de aminoácidos mayor al 50\% entre varios miembros del género Flavivirus. Como otros Flavivirus, la proteasa del virus del dengue es una serina proteasa con una tríada catalítica (His51, Asp75, and Ser135) y corresponde a la súper familia tripsina. La actividad de la proteasa es dependiente de la asociación con la región hidrofílica de 40 aminoácidos de la proteína NS2B, la cual actúa como cofactor y participa activamente en la formación de los sub-bolsillos S2 y S3 en el sitio activo de la proteasa. Ambas proteasas, viral y del huésped, son necesarias para el procesamiento de la poli proteína viral, la cual es esencial para la replicación viral y el ensamble de viriones, en el ensamblaje intervienen enzimas codificadas en el ácido nucleico del virus $[12,13]$. Los inhibidores clásicos de las serina proteasas no son efectivos o tienen baja efectividad contra la proteasa NS3 del virus del dengue. A pesar de los esfuerzos para encontrar inhibidores para las diferentes proteasas de los Flavivirus, usando estrategias tales como librerías de cribado, péptidos miméticos y cribado virtual, hasta la fecha, ningún compuesto ha mostrado tener propiedades apropiadas para el desarrollo de un medicamento. Esto se debe a que los inhibidores se unen débilmente a la enzima, dando lugar a uniones débiles y no covalentes que generan un cambio en la conformación estructural de la enzima que repercute en el sitio activo, afectando así la actividad enzimática [12]. Aldehídos tripéptidos, compuestos basados en antraceno, ciclopéptidos análogos de kalata-Bl y $\alpha$-cetoamidas han sido reportados recientemente como inhibidores de la serina proteasa NS2B/NS3 del virus del dengue [13-15].

\section{METODOLOGÍA}

\section{DATOS COMPUTACIONALES}

Obtención, refinación y optimización de la estructura tridimensional de la proteasa NS2B/NS3

Inicialmente, la estructura de la proteasaNS2B/NS3 del virus del dengue tipo 2 fue descargada de la base de datos del Protein Data Bank (PDB) [16]. La estructura cristalina, con código de acceso pdb 2FOM, posee una resolución de $1.5 \AA$.

La estructura 2FOM, fue refinada mediante la adición de hidrógenos y eliminación de ligandos utilizando el programa Discovery Studio 2.5. Posteriormente, los hidrógenos adicionados a la estructura fueron optimizados utilizando el paquete de programas Sybyl7.3 [17], y los siguientes parámetros: método gradiente conjugado, gradiente de 0.025 $\mathrm{kcal} / \mathrm{mol} \AA$, máximo de 100 iteraciones, campo de fuerza Tripos, cargas de Gasteiger-Huckel y constante dieléctrica de 1.0 .

\section{Búsqueda, selección, construcción y optimización de ligandos}

Se seleccionaron 15 compuestos reportados en 2011 por Schüller et al [13]. Para el estudio de modelado molecular con docking y 3D-QSAR. Los compuestos corresponden a una series de inhibidores de la proteasa NS2B/NS3 del virus del dengue con actividad inhibitoria in vitro comprobada. Los compuestos con su correspondiente estructura y actividad inhibitoria se detallan en la Tabla 1. Las estructuras de los 15 ligandos (inhibidores de la proteasa NS2B/NS3) fueron dibujadas y optimizadas utilizando los programas Gaussview 4.1 y Gaussian 09 [18] respectivamente. Las estructuras de los ligandos fueron optimizadas empleando el método semiempírico AM1 [19]. 
Tabla 1. Estructuras y actividades inhibitorias de los compuestos reportados como inhibidores del complejo proteico NS2B/NS3 del virus del dengue tipo 2 [12]. Table 1. Structures and inhibitory activities of compounds reported as inhibitors of the NS2B/NS3 protein complex of dengue virus type 2 [12].

\begin{tabular}{|c|c|c|}
\hline No. & Estructura & Actividad inhibitoria \\
\hline 1 & & $\operatorname{IC} 50(\mu \mathrm{M})=9.5 \pm 0.21$ \\
\hline 2 & & $\operatorname{IC} 50(\mu \mathrm{M})=6.7 \pm 1.1$ \\
\hline 3 & & $\operatorname{IC} 50(\mu \mathrm{M})=167 \pm 47$ \\
\hline 4 & & $\operatorname{IC} 50(\mu \mathrm{M})=201 \pm 33$ \\
\hline 5 & & $\operatorname{IC} 50(\mu \mathrm{M})=58 \pm 7.2$ \\
\hline 6 & & $\operatorname{IC50}(\mu \mathrm{M})=115 \pm 23$ \\
\hline 7 & & $\operatorname{IC} 50(\mu \mathrm{M})=218 \pm 18$ \\
\hline 8 & & $\operatorname{IC} 50(\mu \mathrm{M})=23.4 \pm 1.4$ \\
\hline 9 & & $\operatorname{IC} 50(\mu \mathrm{M})=12.2 \pm 0.38$ \\
\hline 10 & & $\operatorname{IC} 50(\mu \mathrm{M})=11.2 \pm 0.28$ \\
\hline 11 & & $\operatorname{IC} 50(\mu \mathrm{M})=26.7 \pm 0.11$ \\
\hline 12 & & $\operatorname{IC} 50(\mu \mathrm{M})=15.8 \pm 4.5$ \\
\hline 13 & & $\operatorname{IC} 50(\mu \mathrm{M})=127 \pm 2.1$ \\
\hline 14 & & $\operatorname{IC} 50(\mu \mathrm{M})=172 \pm 10$ \\
\hline 15 & & $\operatorname{IC} 50(\mu \mathrm{M})=274 \pm 27$ \\
\hline
\end{tabular}

\section{RESULTADOS Y DISCUSIÓN}

\section{Acoplamiento molecular}

Posteriormente, se llevó a cabo el acoplamiento molecular entre cada uno de los ligandos optimizados y el complejo proteasa NS2B/NS3 refinado. Para este paso se utilizó el programa de docking molecular Autodock 4.2 [20]. Cada uno de los 15 ligandos fue acoplado en el sitio catalítico conformado por los residuos de aminoácido His51, Asp75 y Ser135 de la cadena B del complejo proteasa NS2B/NS3 del virus del dengue tipo 2. Para el docking molecular se utilizó el algoritmo genético. La pose con menor energía de libre de unión, generada del acoplamiento para cada ligando fue escogida como la mejor pose. Los resultados derivados del acoplamiento molecular, para la mejor pose de cada ligando, se muestran en la Tabla 2.

Tabla 2. Energías y datos calculados para la mejor pose en la interacción ligandoproteína en los 15 inhibidores de la proteasa NS2B/NS3 del virus del dengue. Table 2. Energies and data calculated for the best pose in the ligand-protein interaction in the 15 inhibitors of the NS2B/NS3 protease of the dengue virus.

\begin{tabular}{|c|c|c|c|c|c|c|c|c|c|c|}
\hline o. & 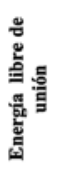 & 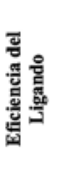 & 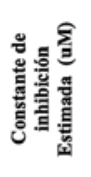 & 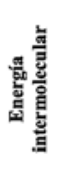 & 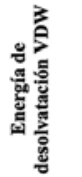 & 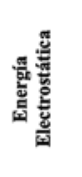 & 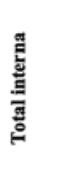 & 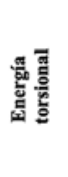 & 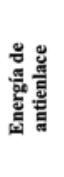 & $\sum_{\Perp}^{\infty}$ \\
\hline 1 & & .07 & 91 & 10. & -10.16 & -0. & -2.2 & 7.46 & 2.24 & 20.49 \\
\hline 2 & .44 & .14 & 102.08 & 11.71 & -10.72 & -0.99 & -2.6 & 6.26 & -2.6 & 21.52 \\
\hline 3 & -4.65 & -0.12 & 388.12 & -11.22 & -9.35 & -1.87 & -0.32 & 6.56 & -0.32 & 22.55 \\
\hline 4 & -6.07 & -0.15 & .69 & -12.33 & -11.01 & -1.32 & -0.76 & 6.26 & -0.76 & 22.06 \\
\hline 5 & -3.69 & -0.11 & 198 & -9.36 & -8.54 & -0.82 & -1.15 & 5.67 & -1.15 & 20.49 \\
\hline 6 & -4.00 & -0.13 & & .97 & -8.18 & -1.79 & -1.42 & 5.97 & -1.42 & 21.79 \\
\hline 7 & -5.30 & -0.15 & 129.47 & -11.27 & -9.85 & -1.42 & -0.48 & 5.97 & -0.48 & 20.01 \\
\hline 8 & -5.40 & -0.12 & & -11.96 & -11.36 & -0.6 & -1 . & 6.56 & -1.37 & 20.28 \\
\hline 9 & -4.40 & -0.10 & 590.76 & -11.27 & -10.38 & -0.89 & -1.46 & 6.86 & -1.46 & 20.49 \\
\hline 10 & -4.95 & -0.12 & 234.28 & -11.52 & -11.13 & -0.39 & -0.57 & 6.56 & -0.57 & 19.76 \\
\hline 11 & -6.78 & -0.16 & & -10.06 & -8.92 & -1.14 & -0.65 & 3.28 & -0.65 & 22.01 \\
\hline 12 & -5.64 & -0.14 & & & -11.41 & -0.49 & -1.86 & 6.26 & -1.86 & 20.62 \\
\hline 13 & -4.52 & -0.12 & 483.12 & -10.79 & -10.00 & -0.79 & -2.28 & 6.26 & -2.28 & 19.8 \\
\hline 14 & -3.98 & -0.12 & & & -9.45 & -0.79 & -0.58 & 6.26 & -0.58 & 20.45 \\
\hline 15 & -4.26 & -0.12 & 752.51 & -10.53 & -8.55 & -1.97 & -1.39 & 6.26 & -1.39 & 21.25 \\
\hline
\end{tabular}

Las interacciones de tipo puente de hidrógeno entre los residuos de aminoácido de la proteasa NS2B/NS3 y los inhibidores se detallan en la Tabla 3.

Tabla 3. Número de interacciones puente de hidrógeno entre cada inhibidor y los residuos de aminoácido del complejo proteasa NS2B/NS3.

Table 3. Number of hydrogen bridge interactions between each inhibitor and the amino acid residues of the NS2B/NS3 protease complex

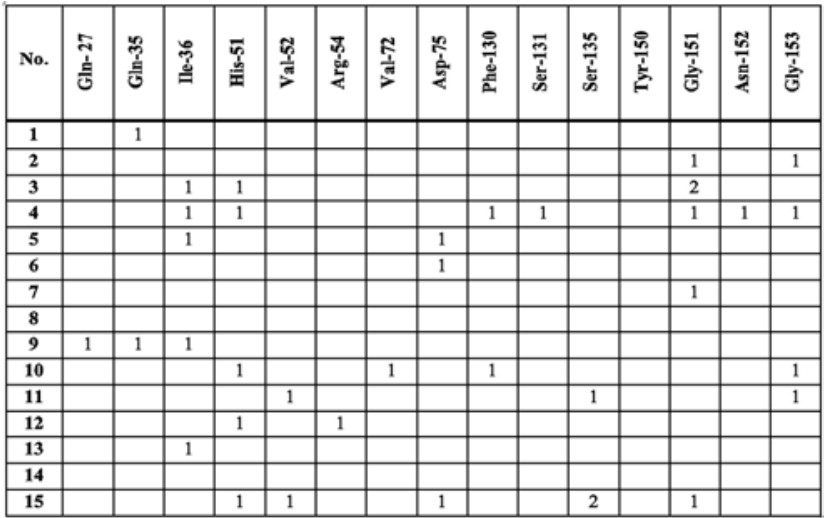


Por otro lado, las interacciones de Van der Waals entre los residuos de aminoácido de la proteasa NS2B/NS3 y los inhibidores se detallan en la Tabla 4.

Tabla 4. Interacciones de Van der Waals entre cada inhibidor y los residuos de aminoácido del complejo proteasa NS2B/NS3. Table 4. Van der Waals interactions between each inhibitor and the amino acid residues of the NS2B/NS3 protease complex.

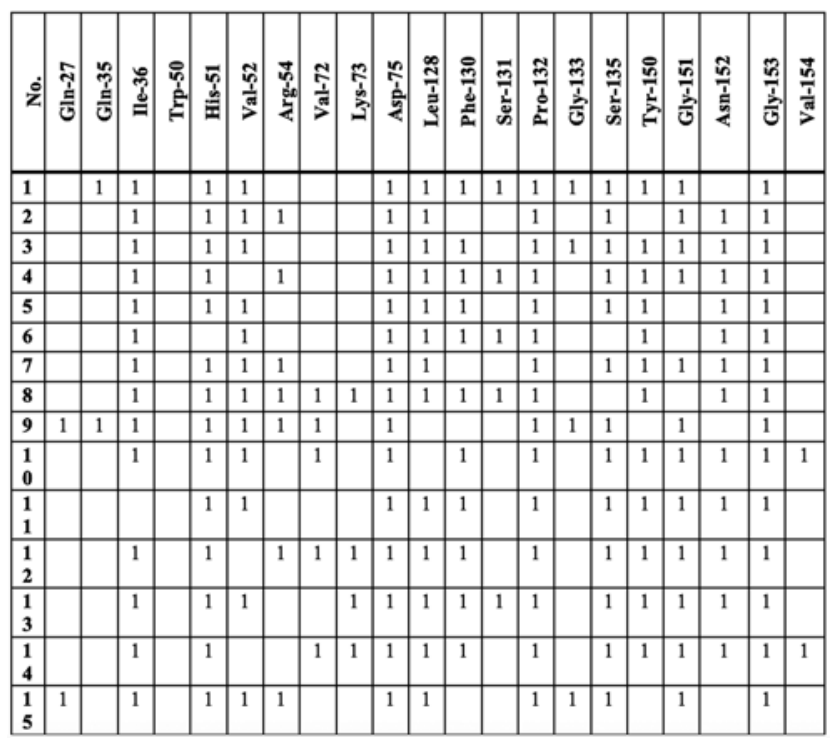

\section{Datos para 3D QSAR}

Para realizar los cálculos3D QSAR, tomamos de la Tabla 1 los 15 péptidos que allí se muestran. Las actividades inhibitorias que se reportan de estos péptidos contra la proteasa NS2B/NS3 del virus del dengue fueron medidas in vitro para la serie de compuestos objeto de este estudio (ver Tabla 1) [12]. Se descargó de la base de datos ProteinData Bank [16] la estructura tridimensional del Benzoil-nKRR-H (péptido 1) acoplado a dicha proteasa, este archivo tiene como código de descarga 2FP7. Con la ayuda del programa Discovery Studios 5.0 se extrajo la estructura de este péptido el cual se muestra en la Figura 1. Ya que la geometría del péptido 1 mostrada en la Figura 1 corresponde al acoplamiento de este péptido con la proteasa NS2B/NS3 del virus del dengue, consideramos que los demás péptidos deben tener esta geometría para poder acoplarse a dicha proteína; por lo cual decidimos modificar esta estructura para obtener un molde en la construcción de los 14 péptidos restantes. El molde obtenido es el que se muestra en la Figura 2, en el cual se eliminó el grupo Benzoil-Norluesina presente en el péptido 1 (Figura 1) y solo se dejó la secuencia Lisina-Argenina.

\section{Alineamiento}

La construcción de los péptidos en 3D se realizó con el programa GuassView4.1. Todas las estructuras excepto la del péptido 1 fueron construidas a partir de la estructura mostrada en la Figura 2, ya que entendemos que estas serían las conformaciones más probables. Una vez dibujadas las moléculas, fueron optimizadas utilizando la metodología ONION implementada en el programa Gaussian, [18] en el cual establecimos como LowLayer (PM3) [18] a la parte de cada molécula que no queríamos que variara con la optimización y HightLayer (DFT B3LYP, 6-31G) [21] a la parte de cada molécula que si queríamos que cambiara. Ya con los péptidos debidamente optimizados y haciendo uso del programa Sybyl $X$ (2.0) [17] creamos una base de datos con todos los péptidos, la cual fue debidamente alineada y de la cual obtuvimos los diferentes modelos 3D QSAR.

Figura 1. Péptido 1. Bz-nKRR-H. Figure 1. Peptide 1. Bz-nKRR-H.

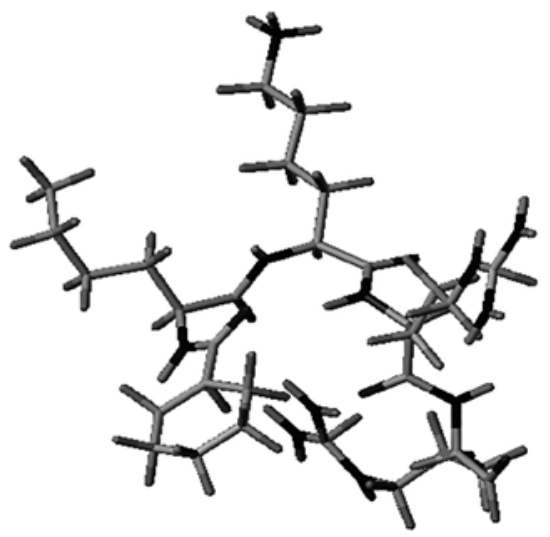

Figura 2. Molde para la construcción de los péptidos en el cual se eliminó el grupo Benzoil-Norleusina presente en el péptido 1 (Fig. 1) y solo se dejó la secuencia Lisina-Argenina-Argenina.

Figure 2. Mold for the construction of the peptides in which the BenzoylNorleusin group present in peptide 1 was eliminated (Fig. 1) and only the sequence Lysine-Argenine-Argenine was left.

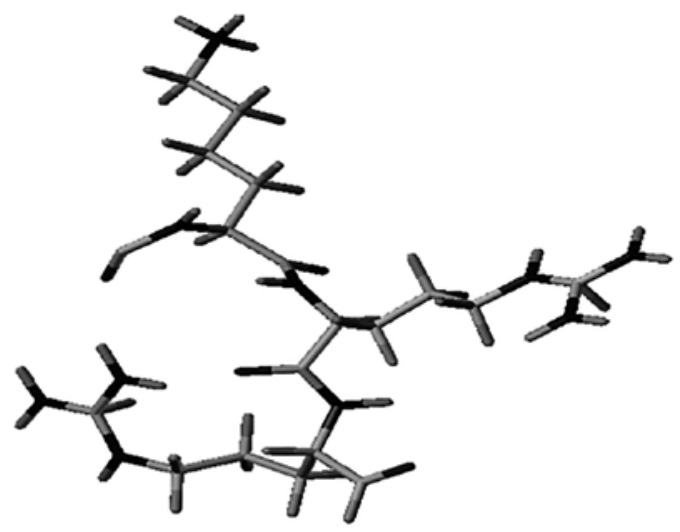

\section{Analisis CoMFA}

Las moléculas alineadas se colocaron en una celda de la malla 3D de tal manera que el conjunto completo estuviera incluido en ella. Las energías de los campos estéricos (Potencial de Lennard-Jones 6-12) y el campo electroestático (Potencial de Coulomb) fueron calculadas utilizando carbonos $s p^{3}$ como sonda y se aplicó el campo de fuerza estándar 
TRIPOS. La función dieléctrica se seleccionó como dependiente de la distancia. Las energías se truncaron a $\pm 30 \mathrm{kcal}$ $\mathrm{mol}^{-1}$.

En todos los alineamientos que se probaron se escogió como molécula platilla el péptido 2 (Tabla 1), para el cual construimos la estructura que se muestra en la Figura 3.

Figura 3. Estructura molecular del péptido 2. Plantilla para los distintos alineamientos de las primeras 15 moléculas mostradas en la Tabla 1. Figure 3. Molecular structure of peptide 2. Template for the different alignments of the first 15 molecules shown in Table 1.

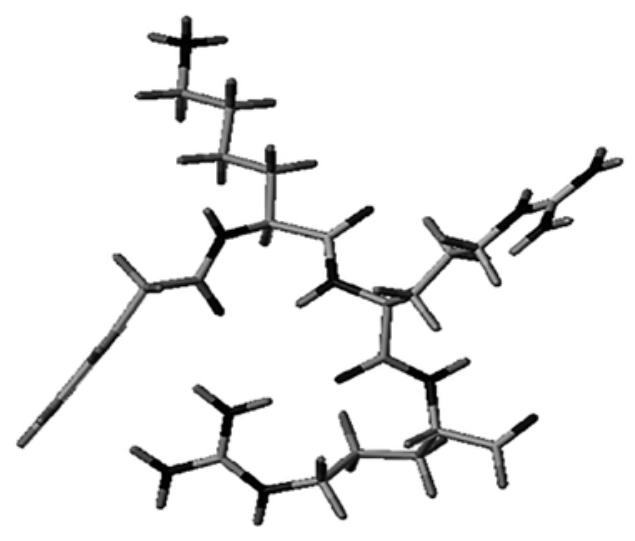

El mejor modelo 3D QSAR que se obtuvo, fue el hecho a partir del alineamiento que se muestra en la Figura 4, la cual muestra como los péptidos quedan muy bien alineados en la mayoría de las zonas, especialmente sobre la Argenina terminal de cada péptido.

Figura 4. Alineamiento basado en la estructura molecular del péptido 2. Se observa que las moléculas quedan muy bien alineadas especialmente sobre la última Argenina de cada péptido.

Figure 4. Alignment based on the molecular structure of peptide 2. It is observed that the molecules are very well aligned especially on the last Argenin of each peptide.

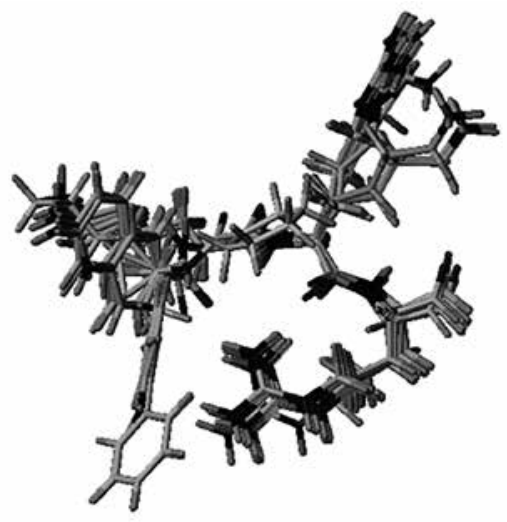

\section{Analisis PLS}

Los descriptores CoMFA se usaron como variable independiente y los valores de $\mathrm{pIC}_{50}$ se utilizaron como variable dependiente en la regresión por mínimos cuadrados PLS en la deducción del modelo 3D QSAR. La eficiencia del método se calcula usando la validación cruzada Leave-one-out (LOO), [22] valor que se conoce como $\mathrm{Q}^{2}$.

En la Figura 5 se grafican los valores reportados en la referencia 14 versus los generados por el programa CoMFA. Como se puede ver los valores reportados del anaisis PLS fueron un $\mathrm{Q}^{2}=0.550$ y $\mathrm{R}^{2}=0.984$.

Figura 5. Grafica de $\mathrm{plC} \mathrm{C}_{50}$ experimental vs $\mathrm{plC}_{50}$ predicha por el CoMFA. Figure 5. Graph of plC ${ }_{50}^{5}$ experimental vs $\mathrm{plC}_{50}^{50}$ predicted by CoMFA.

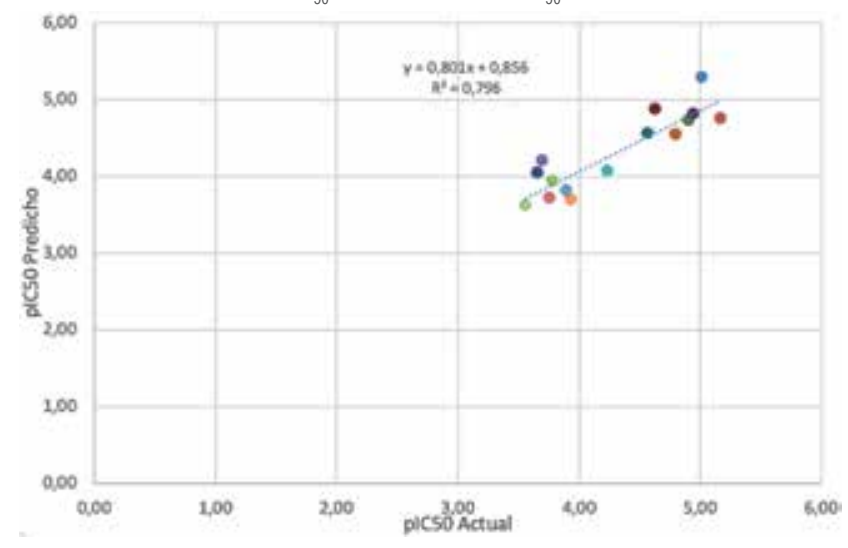

Los valores obtenidos del anaisis PLS fueron un $\mathrm{Q}^{2}=0.550$ y $\mathrm{R}^{2}=0.984$. Los campos generados por el 3D-Qsar se muesran en la Figura 5.

Tabla 5. Valores experimentales y predichos de actividadusando el programa CoMFA. Table 5. Experimental and predicted activity values using the CoMFA program.

\begin{tabular}{c|c|c}
\hline Peptido & plC50 Actual & pIC50 CoMFA \\
\hline 1 & 5,02 & 5,29 \\
2 & 5,17 & 4,75 \\
3 & 3,78 & 3,93 \\
4 & 3,7 & 4,2 \\
5 & 4,24 & 4,07 \\
6 & 3,94 & 3,69 \\
7 & 3,66 & 4,04 \\
8 & 4,63 & 4,87 \\
9 & 4,91 & 4,72 \\
10 & 4,95 & 4,81 \\
11 & 4,57 & 4,55 \\
12 & 4,8 & 4,54 \\
13 & 3,9 & 3,81 \\
14 & 3,76 & 3,71 \\
15 & 3,56 & 3,61 \\
\hline
\end{tabular}


Figura 6. Campo estérico generado por el 3D-QSAR de

15 péptidos alineados y los campos que los rodean.

Figure 6 . Steric field generated by the 3D-QSAR of

15 aligned peptides and the fields that surround them.

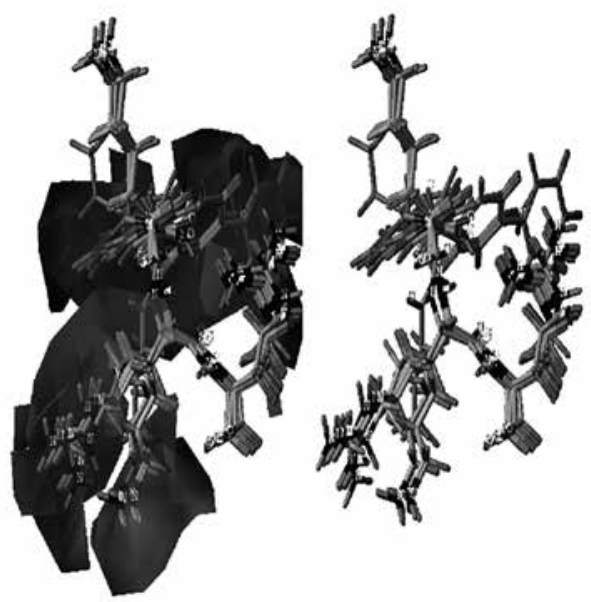

\section{CONCLUSIONES}

Las regiones verdes mostradas en los mapas indican la favorabilidad a modificaciones que incluyan grupos más voluminosos que los que allí se muestran. Sin embargo, los grupos que sustituyan a los presentes deben tener las mismas características químicas que a quienes estén reemplazando. Los péptidos tienen una secuencia R-KRR-H o R-KKR-H. Se puede ver que estas zonas verdes están rodeando a los sustituyentes R, lo que nos indica que estos deben ser más voluminosos. Esto se puede ver en la Tabla 1, donde se muestra que los péptidos que tienen sustituyentes más grandes en tamaño poseen actividades inhibitorias más altas que aquellos que no tienen grupos grandes. Si tomamos en consideración los péptidos 7 y 15 , podemos ver que estos no tienen sustituyentes derivados del benceno y poseen actividades muy bajas; para estos péptidos el modelo 3D QSAR predijo una actividad inhibitoria mayor, como se muestra en la Tabla 5 , si se realizaran las mejoras estructurales pertinentes. Hay que tener en cuenta que, de los 15 péptidos tomados para realizar el 3D QSAR, cinco de ellos (péptido: 5; 6; 7; 14 y 15) no tienen sustituyentes derivados del benceno, por el contrario tienen cadenas cortas de átomos de carbono, por tanto las modificaciones a realizar sobre ellos será reemplazar estas cadenas de carbonos por grupos aromáticos como los fenilos. Por otra parte, para mejorar la actividad de aquellos péptidos que tienen una actividad inhibitoria alta y que tienen derivados del benceno como grupo $\mathrm{R}$, una buena alternativa será probar con el antraceno y algunos de sus derivados, ya que presentan las mismas características químicas y es un compuesto más voluminoso que los presentes en la mayoría de los péptidos.

\section{REFERENCIAS}

[1] G. Kouri, M. Guzman. "Dengue and dengue Hemorrhagic Fever in the Americas: Lessons and Challenges". Journal of Clinical Virology, 27(1), 1-13, 2003.

[2] A. Ramirez, Z. Moros, G. Comach, J. Zambrano, L. Bravo, B. Pinto, S Vielma, J. Cardier, F. Liprandi. "Evaluation of dengue NSl Antigen Detection Tests with Acute Sera from Patients Infected with dengue Virus in Venezuela". DiagnosticMicrobiology and Infectious Disease, 65(3), 247-253, 2003. b. J.C. Lin, S.C. Lin, W.Y, Chen, Y.T. Yen, C.W. Lai, M.H. Tao, Y.L. Lin, S.C. Miaw, B. Wu-Hsieh. "Dengue Viral Protease Interaction with NF-囚B Inhibitor $\mathbb{Q} \otimes$ Results in Endothelial Cell Apoptosis and Hemorrhage Development". The Journal of Immunology, 193(3), 1258-1267, 2014

[3] E. Kellya, B. Purib, W. Sun, B. Falgoutc. "Identification of Mutations in a Candidate dengue 4 Vaccine Strain 341750 PDK20 and Construction of a Full-Length cDNA Clone of the PDK20 Vaccine Candidate". Vaccine, 28(17), 3030-3037, 2010

[4] S. Murrel, S. Wu, M. Butler. "Review of dengue Virus and the Development of a Vaccine". Biotechnology Advances, 29(2), 239-247, 2011.

[5] World Health organization, W.P.R. Dengue and dengue Hemorrhagic Fever (Disponible Online).http://wwww who.int/mediacentrelfactsheets/ fs117/en/2009.b.http://www.who.int/mediacentrelfactsheets/fs1 17/en/. Marzo de 2014

[6] G. Kouri, M. Guzman. "Dengue diagnosis, Advances and Challenges", InternationalJournal of Infectious Diseases, 8(2), 69-80, 2004.

[7] W. McBride, H. Bielefeldt-Ohmann. "Dengue Viral Infections; Pathogenesis and Epidemiology". Microbes and Infection, 2(9), 1041-1050, 2000 .

[8] M. Bollati, K. Alvarez, R. Assenberg, C. Barontid, B. Canard, S. Cooke, B. Coutard, E. Decroly, X. Lamballeried, E. Gouldd, G. Grardd. "Structure and Functionality in Flavivirus NS-Proteins: Perspectives for Drug Design”. Antiviral Research, 87(2), 125-148, 2010.

[9] J. Lescara, D. Luo, T. Xu, A. Sampath, S. Pheng, B. Canard, S. Vasudevand. "Towards the Design of Antiviral Inhibitors against Flaviviruses: The Case for the Multifunctional NS3 Protein from dengue Virus as a Target". Antiviral Research, 80(2), 94-101, 2008.

[10] P. Prusis, M. Lapins, S. Yahorava, R. Petrovska, P. Niyomrattanakit, G. Katzenmeier, J. Wikberg. "Proteochemometrics analysis of substrate interactions with dengue virus NS3 proteases". Bioorganic E Medicinal Chemistry, 16(20), 9369-9377, 2008.

[11] C.C. Yang, Y.C. Hsieh, S.J.; Lee, S.-H. Wu, C.L. Liao, C-H. Tsao, Y-S. Chao, J.H. Chern, C.-P. Wu, A. Yueh. "Novel dengue Virus-Specific NS2B/NS3 Protease Inhibitor, BP2109, Discovered by a HighThroughput Screening Assay". Antimicrobial Agents Chemotherapy, 55(1), 229-238, 2011.

[12] C. Noble, Y. Chen, H. Dong, F. Gu, S. Pheng, W. Schul, Q. Wang, P. Shi. "Strategies for Development of dengue Virus Inhibitors". Antiviral Research, 85(3), 450-462, 2010.

[13] A. Schüller, Z. Yin, B. Chia, D. Doan, H. Kim, L. Shang, T. Peng, J. Hill, S. Vasudevan. "Tripeptide Inhibitors of dengue and West Nile Virus NS2B-NS3 Protease”. Antiviral Research; 92(1), 96-101, 2011.

[14] Y. Gao, T. Cui, Y. Lam. "Synthesis and Disulfide Bond Connectivity-Activity Studies of a Kalata B1-inspired Cyclopeptide against dengue NS2B-NS3 Protease". Bioorganic E Medicinal Chemistry, 18(3), 1331-1336, 2010

[15] "Complejo Proteasa NS2B/NS3 del virus del denguecon BenzoilnKRR-H”, (en línea). Enero 2006[24 septiembre 13]. Disponible en: https://wow w.rcsb.org. 
[16] Protein Data Bank (PDB, http://www.rsch.org/pdb

[17] SYBYL 7.3. Tripos International, 1699 South Hanley Rd., St. Louis, Missouri, 63144, USA. URL: http://wwww.tripos.com

[18] Gaussian 09, Revision B.02. Gaussian, Inc., Pittsburgh, PA, 2009.

[19] J. Michael, J. S. Dewar and Donn M. Storch. "Development and use of quantum molecular models. 75. Comparative tests of theoretical procedures for studying chemical reactions". Journal of the American Chemical Society, 107(13), 3898-3902, 1985.

[20] G. Morris, R. Huey, W. Lindstrom, M. Sanner, R. Belew, D. Goodsell, A. Olson. "Autodock4 and AutoDockTools4: automated docking with selective receptor flexiblity." Journal of Computational Chemistry, 30(16), 2785-2791, 2009.

[21] A. Becke. "Density-functional thermochemistry. III. The role of exact exchange". Journal Chemistry Physics. 98(7), 5648-5652, 1993. b. C. Lee, W. Yang, R. Parr, Physical Review B. 37(2); 785-789, 1998.

[22] P. Devijver, J. Kittler, Pattern Recognition: A Statistical Approach, Prentice-Hall, Londres, 1982. 